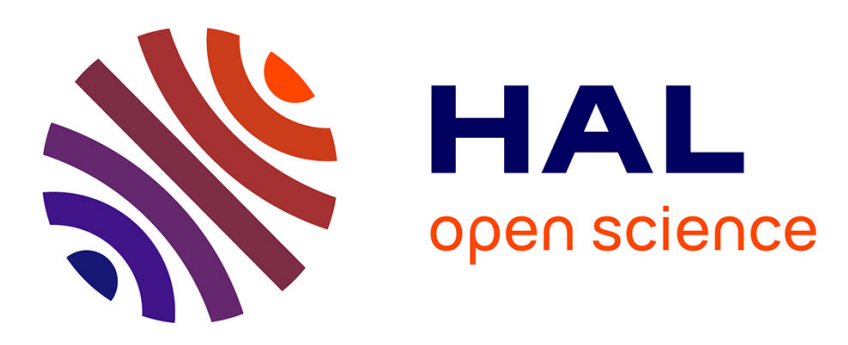

\title{
Bulk wave velocities in cortical bone reflect porosity and compression strength
}

Laura Peralta, Maeztu Redin, Fan Fan, Xiran Cai, Pascal Laugier, Johannes

Schneider, Kay Raum, Quentin Grimal

\section{- To cite this version:}

Laura Peralta, Maeztu Redin, Fan Fan, Xiran Cai, Pascal Laugier, et al.. Bulk wave velocities in cortical bone reflect porosity and compression strength. Ultrasound in Medicine \& Biology, 2021, 47 (3), pp.799-808. 10.1016/j.ultrasmedbio.2020.11.012 . hal-03145788

\author{
HAL Id: hal-03145788 \\ https://hal.science/hal-03145788
}

Submitted on 18 Feb 2021

HAL is a multi-disciplinary open access archive for the deposit and dissemination of scientific research documents, whether they are published or not. The documents may come from teaching and research institutions in France or abroad, or from public or private research centers.
L'archive ouverte pluridisciplinaire HAL, est destinée au dépôt et à la diffusion de documents scientifiques de niveau recherche, publiés ou non, émanant des établissements d'enseignement et de recherche français ou étrangers, des laboratoires publics ou privés. 


\title{
Bulk wave velocities in cortical bone reflect porosity and compression strength
}

\author{
Laura Peralta ${ }^{\mathrm{a}, \mathrm{b}, *}$, Juan Deyo Maeztu Redin ${ }^{\mathrm{a}}$, Fan Fan ${ }^{\mathrm{a}, \mathrm{c}}$, Xiran Cai ${ }^{\mathrm{a}}$, \\ Pascal Laugier $^{\mathrm{a}}$, Johannes Schneider ${ }^{\mathrm{d}}$, Kay Raum ${ }^{\mathrm{d}}$, Quentin Grimal ${ }^{\mathrm{a}}$ \\ ${ }^{a}$ Sorbonne Université, INSERM, CNRS, Laboratoire d'Imagerie Biomédicale, LIB, \\ F-75006 Paris, France \\ ${ }^{b}$ Department of Biomedical Engineering, School of Biomedical Engineering $\&$ Imaging \\ Sciences, King's College London, London, UK \\ ${ }^{c}$ Beijing Advanced Innovation Center for Biomedical Engineering, School of Biological \\ Science and Medical Engineering, Beihang University, 100083, Beijing, China \\ ${ }^{d}$ Berlin-Brandenburg School for Regenerative Therapies, Charité - Universitätsmedizin \\ Berlin, 13353 Berlin, Germany
}

\begin{abstract}
The goal of this study is to evaluate whether ultrasonic velocities in cortical bone can be considered as a proxy for mechanical quality of cortical bone tissue reflected by porosity and compression strength. Micro-computed tomography, compression mechanical testing, and resonant ultrasound spectroscopy were used to assess, respectively porosity, strength, and velocity of bulk waves of both shear and longitudinal polarisations propagating along and perpendicular to osteons, in 92 cortical bone specimens from tibia and femur of elderly human donors. All velocities were significantly associated with strength ( $r=0.65$ to 0.83$)$ and porosity $(r=-0.64$ to -0.77$)$. Roughly, according to linear regression models, a decrease in velocity of $100 \mathrm{~ms}^{-1}$ cor-
\end{abstract}

\footnotetext{
*Corresponding Author: Laura Peralta, Department of Biomedical Engineering, School of Biomedical Engineering \& Imaging Sciences, King's College London, St Thomas' Hospital, SE1 7EH, London, UK; Email: laura.peralta_pereira@kcl.ac.uk
} 
responded to a loss of $20 \mathrm{MPa}$ of strength (which is approximately $10 \%$ of the largest strength value) and to a porosity increase of 5\%. These results provide a rationale for the in vivo measurement of one or several velocities for the diagnosis of bone fragility.

Keywords: resonant ultrasound spectroscopy, bone, strength, velocity, porosity, shear, anisotropy 


\section{Introduction}

2

Aging and primary or secondary osteoporosis are associated with an alteration of the mechanical quality of bones, leading to atraumatic fractures which reduce the quality of life and increase mortality. This is a major global health problem as nine million fragility fractures occur annually worldwide (Cooper and Ferrari, 2017). In practice, fracture risk is assessed based on clinical factors and, in the standard approach, bone mineral density (BMD) measured with dual-energy X-ray absorptiometry (DXA). However, this approach has strong limitations. DXA is an ionizing method and has a low sensitivity to identify individuals who sustain fragility fractures (Siris et al., 2004; Briot et al., 2013).

Ultrasound (US) methods have been developed as an alternative to DXA to provide a non-ionizing, portable, and affordable diagnostic tool for osteoporosis (Laugier and Haïat, 2011; Raum et al., 2014). Since cortical bone plays an important role in bone resistance (Mayhew et al., 2005; Holzer et al., 2009), and because a large part of bone loss arises from the cortical compartment (Zebaze et al., 2010), several US approaches have been specifically designed to assess cortical bone (Karjalainen et al., 2008; Sai et al., 2010; Minonzio et al., 2019; Renaud et al., 2018; Nguyen Minh et al., 2020; Grimal and Laugier, 2019). These approaches aim at evaluating cortical bone thickness or material properties (e.g., mass density, elasticity, bulk wave velocities), which are dramatically altered with bone pathologies.

Mechanical properties of cortical bone tissue are essentially determined by the vascular pore network (volume fraction of pores or, shortly, the porosity, and microarchitecture) and the properties of the extravascular mineralized 
matrix surrounding pores (Mirzaali et al., 2016; Cai et al., 2019). Previous studies on cortical bone have shown that US velocities depend on porosity and matrix properties (Raum et al., 2005; Grondin et al., 2012; Mathieu et al., 2013; Eneh et al., 2016). However, to which extent US velocities of both shear and longitudinal polarisations relate to cortical bone mechanical resistance is still largely unknown.

This study aims to evaluate whether US bulk wave velocities in cortical bone can be considered as proxy for mechanical quality of bone tissue reflected by porosity and compression strength. One originality of the study is that US waves of both shear and longitudinal polarisations propagating in different anatomical directions are considered.

The elastic behaviour of cortical bone is most often described using an orthotropic or a transversely isotropic framework (Espinoza Orías et al., 2009; Granke et al., 2011). Anisotropy is due to the preferential alignement of osteons (and the Haversian canal at their center) along the diaphysis and the preferential arrangement of mineralized collagen fibers along the axis of the osteons. It follows that bulk wave velocities depend on the orientation of the wave vector relative to osteons. To determine US velocities of both shear and longitudinal waves propagating in any anatomical direction, in this study, the entire stiffness tensor of bone specimens was measured by resonant ultrasound spectroscopy (RUS) (Bernard et al., 2013).

Cortical bone material resistance to fracture is usually characterized ex vivo by measurement of strength (i.e., ultimate stress in a compression or traction test) or toughness (i.e., resistance to crack propagation) (Zimmermann et al., 2015). In this study, compressive ultimate stress (strength) was 
assessed. Porosity was also assessed as it is an important determinant of bone mechanical quality, and is recognized as a fracture risk factor (Ahmed et al., 2015; Bala et al., 2015; Zebaze et al., 2016).

Quantitative ultrasonography, based on the velocity of transmission of an US signal inside the bone, has been widely used for the investigation of bone status and previous studies have reported its correlation with bone strength (Lee et al., 1997; Hudelmaier et al., 2004). However, to the best of our knowledge, there is no previous study of a direct comparison of material strength in human cortical bone and shear and longitudinal bulk wave velocities along the principal material axes (along and perpendicular to osteons). The data presented here provide a rationale for the in vivo measurement of one or several US velocities as a proxy for bone tissue mechanical resistance to complement US diagnosis of bone fragility.

\section{Materials and Methods}

\section{Specimens}

Left and right femora and left tibiae from 19 human cadavers were provided by the Institute of Anatomy, University of Lübeck. The scientific use of human tissue from body donors is permitted by the German law "Gesetz über das Leichen-, Bestattungsund Friedhofswesen des Landes Schleswig- HolsteinAbschnitt II, §9 (Leichenöffnung, anatomisch)" from 04.02.2005. All subjects had given consent for the scientific use of their bodies. Among the donors, 13 were females (ages 69-94 years, mean \pm standard deviation $=82.7 \pm 8.4$ years) and 6 were males (ages 70-94 years, $82.2 \pm 10.1$ years). No other information on donors was available. The fresh material was frozen and stored 
at $-20^{\circ} \mathrm{C}$ until and between experiments.

For each bone, one cross-section of approximately $20 \mathrm{~mm}$ thickness and perpendicular to the bone axis was extracted using a precision band saw (EXACT GmbH, Remscheid, Germany). For each tibia, the cross-section was cut from the midshaft and at $19.5 \pm 3.8 \mathrm{~cm}$ away from the proximal end of the bone (Iori et al., 2019). For each femur, the cross-section was extracted from the diaphysis at $80 \mathrm{~mm}$ below the lesser trochanter (Iori et al., 2020). Then, from each cross-section, one or two rectangular parallelepiped shaped specimens were prepared using a diamond wafering blade saw (Isomet 4000, Buehler GmbH, Düsseldorf, Germany) as described in (Cai et al., 2019), see Figure 1. For each tibia, one specimen was obtained from the centre of the medial face of the bone. No specimen was extracted from three tibiae which cortical thickness was too thin. For each femur, two specimens were obtained from the anterior and lateral anatomical quadrants. The nominal dimensions of the specimens were $3 \mathrm{~mm} \times 4 \mathrm{~mm} \times 5 \mathrm{~mm}$ for femur, and 2 $\mathrm{mm} \times 3 \mathrm{~mm} \times 4 \mathrm{~mm}$ for tibia, in radial (axis 1), circumferential (axis 2) and axial (axis 3, along the diaphysis) directions, respectively, defined by the anatomical shape of the bone (see Figure 1). Note that direction 3 is also the main direction of osteons and their cylindrical canal. A total of 16 and 76 rectangular parallelepiped shaped specimens, from tibia and femur bones, respectively, were prepared. The dimensions $($ mean $\pm \mathrm{SD})$ of the prepared specimens from tibia bones were $2.00 \pm 0.24 \mathrm{~mm}$ (axis 1), $3.10 \pm 0.28 \mathrm{~mm}$ (axis 2 ), and $4.14 \pm 0.27 \mathrm{~mm}$ (axis 3), while the dimensions of the femur specimens were $2.78 \pm 0.39 \mathrm{~mm}$ (axis 1), $3.99 \pm 0.39 \mathrm{~mm}$ (axis 2), and $4.81 \pm 0.38 \mathrm{~mm}$ (axis 3). 
The mass density of each specimen was derived from the averaged values of four mass (Sartorius CPA224s, precision: $0.1 \mathrm{mg}$ ) and dimensions measurements (Mitutoyo Coolant Proof Caliper 500-606, precision: $0.01 \mathrm{~mm}$ ).

Errors on the geometrical shape of the samples following this sample preparation protocol were measured in a previous study on femur specimens, (Cai et al., 2017), where the deviations from ideal perpendicularity and parallelism were $-0.07^{\circ} \pm 0.85$ and $0.30^{\circ} \pm 0.78$, respectively.

Note that another set of similar rectangular parallelepiped shaped specimens from the right tibiae of the same donors were used in another study documenting elastic coefficients based on RUS measurements and their relationship with apparent density (Bernard et al., 2016).

\section{Ultrasonic velocity measurements by resonant ultrasound spectroscopy}

Resonant ultrasound spectroscopy was used to measure the velocity of shear and longitudinal bulk waves along the principal material axis (i.e. along and perpendicular to osteons) of the bone specimens (Migliori and Sarrao, 1997). Velocities along other material directions may be calculated from the stiffness tensor provided in the supplemental material (Auld, 1975). Note that elastic coefficients or velocities can alternatively be measured by the traditional pulse transmission method. A previous empirical study showed that the latter and RUS yield the same elasticity and bulk wave velocity values (Peralta et al., 2017).

Orthotropic symmetry was assumed for femur specimens because they were obtained away from the mid-diaphysis. Indeed, while at the middiaphysis of the femur, bone material is transversely isotropic (Granke et al., 2011; Orías et al., 2009), it is not strictly the case in the rest of the diaph- 


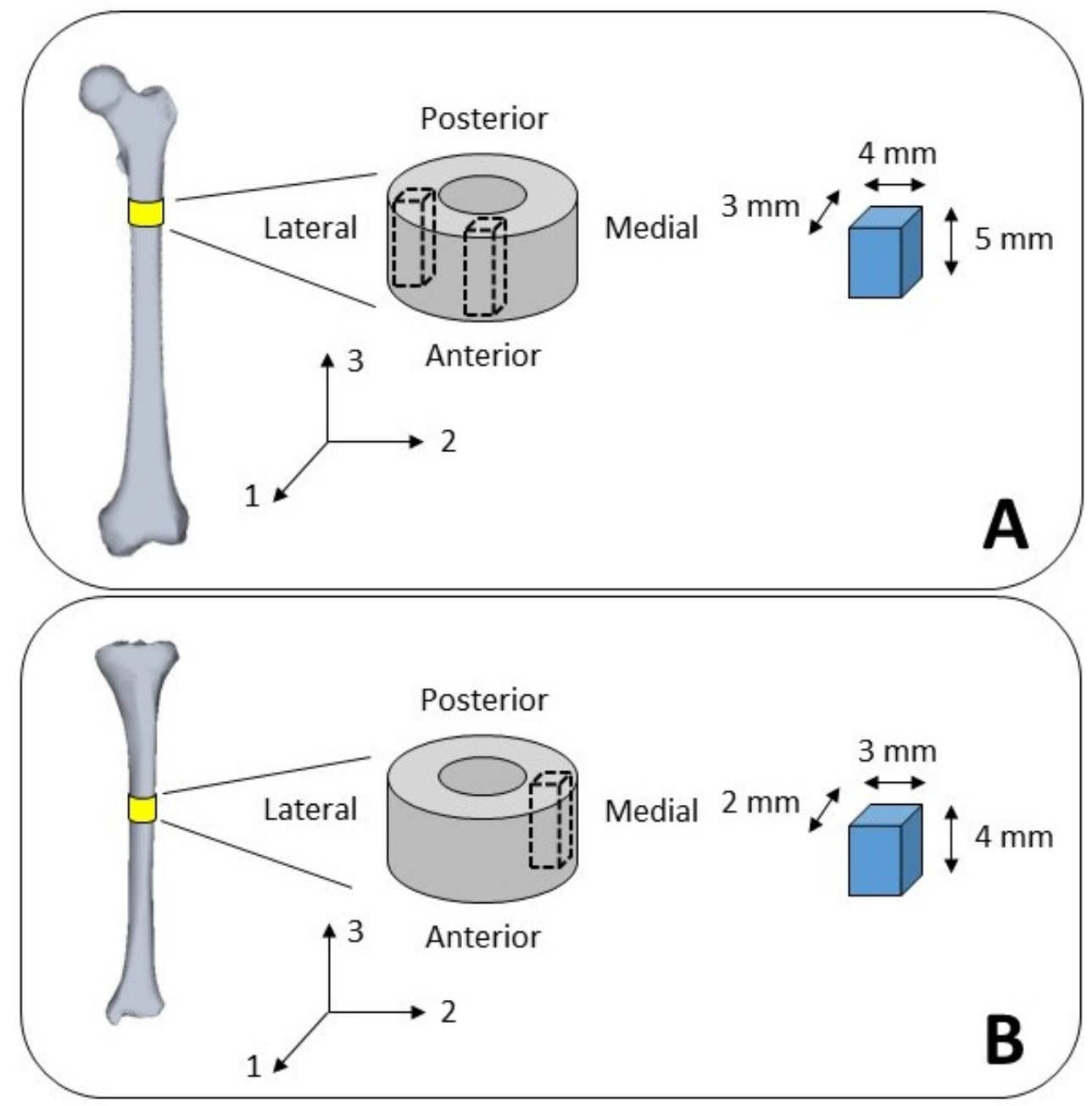

Figure 1: Summary of specimen preparation. (A) Femur specimens. Two rectangular parallelepiped shaped specimens of dimensions $3 \mathrm{~mm} \times 4 \mathrm{~mm} \times 5 \mathrm{~mm}$, in radial (axis 1), circumferential (axis 2) and axial direction (axis 3), extracted from the lateral and anterior anatomical quadrants of a cross-section of the left and right femur shaft. (B) Tibia specimens. One rectangular parallelepiped shaped specimen of dimensions $2 \mathrm{~mm}$ $\times 3 \mathrm{~mm} \times 4 \mathrm{~mm}$, in radial (axis 1), circumferential (axis 2) and axial direction (axis 3), extracted from the medial anatomical quadrant of a cross-section of the left tibia midshaft. 
ysis (Orías et al., 2009). Transversely isotropic symmetry was assumed for tibia specimens, which are isotropic in the plane (1-2) (Bernard et al., 2016; Rho, 1996). The stiffness tensor has nine independent constants $C_{i j}(i j=$ $11 ; 22 ; 33 ; 12 ; 13 ; 23 ; 44 ; 55 ; 66)$ (Voigt notation) for an orthoropic material, and five for a transversely isotropic material $\left(C_{11}=C_{22}, C_{12}=C_{11}-2 C_{66}\right.$, $C_{13}=C_{23}, C_{44}=C_{55}$ ), which correspond to nine and five independent bulk wave velocities, respectively.

RUS measurements were conducted following a procedure extensively described elsewhere (Bernard et al., 2014, 2015). Briefly, bone specimens were placed between two ultrasonic transducers (V154RM, Panametrics, Waltham, MA) to generate and record the frequency response. Specimens were held on opposite corners such that a free boundary condition for vibration can be assumed. A vector network analyzer was used to control the emitted and transmitted signals and measure the ultrasonic frequency response of the bone specimens. The frequency response in the bandwidth $50-800 \mathrm{kHz}$, containing the 30-40 first resonant frequencies, was recorded after being amplified by a broadband charge amplifier (HQA-15 M-10 T, Femto Messtechnik GmbH, Berlin, Germany). Six consecutive measurements were performed on each specimen with the specimen rotated by approximately 15 degrees in between each measurement. This procedure allowed maximizing the number of detectable resonant frequencies. Finally, the bulk wave velocities $V_{i j}$ were calculated by optimizing the misfit function between the experimental and model-predicted resonant frequencies (inverse problem), using the dimensions of each specimen. The optimization problem was formulated in a Bayesian framework (Bernard et al., 2015) which requires to set a prior 
describing the distribution of the velocities. This was constructed based on the stiffness data in Granke et al. (2011) and Bernard et al. (2016) for the femoral and tibial bone specimens, respectively.

The experimental errors of this RUS protocol, associated to the irregularity of a specimen's geometry and to measurement uncertainties of the extracted resonant frequencies have been analyzed in a dedicated study conducted on transversely isotropic femur specimens (Cai et al., 2017). The precision error on stiffness measurements (95\% confidence interval) was estimated to be smaller than $\pm 6 \%$ for longitudinal $\left(C_{i j}, i j=11,33\right)$ and offdiagonal $\left(C_{i j}, i j=12,13\right)$ stiffness constants and $\pm 3 \%$ for the shear stiffness constants $\left(C_{i j}, i j=44,66\right)$. These, after propagating error to velocities, correspond to a precision error smaller than $\pm 3 \%$ for longitudinal waves and $\pm 1.5 \%$ for shear waves. Nevertheless, uncertainties in mass measurements were not considered in (Cai et al., 2017), then it is expected that the error on velocities will be smaller, since velocity measurement by RUS does not require to measure mass, but only resonant frequencies and specimen's dimensions (Leisure and Willis, 1997).

\section{Porosity measurements}

Cortical porosity (Ct.Po) was obtained from micro-computed tomography $(\mu \mathrm{CT})$ scans as described in (Schneider et al., 2019) for a subgroup of specimens due to constrained time before mechanical testing. A total of 12 tibia and 38 femur specimens were scanned. These specimens were chosen to represent the density range of the total specimens prepared. Each specimen was positioned in the $\mu \mathrm{CT}$ system (Skyscan 1172, Bruker MicroCT, Kontich, Belgium) so that the axis 3 was aligned with the rotation axis. A 
source voltage of $80 \mathrm{kV}$, a current of $100 \mu \mathrm{A}$, and steps of $0.3^{\circ}$ over $180^{\circ}$ rotation were used. The exposure time for each frame was $320 \mathrm{~ms}$. Twenty frames were averaged. A 0.5-mm-thick aluminum filter reduced beam hardening artifacts. Three-dimensional images were reconstructed using a filtered back-projection algorithm (NRecon, V1.6.10.4, Skyscan NV, Kontich, Belgium) with $20 \%$ ring artifact correction. For each specimen, a stack of 650 sections was reconstructed with a $1968 \times 1968$ pixel field of view and $7.4 \mu \mathrm{m}$ isotropic voxel size. Further post-processing was performed using the software CTan (V1.16.1.0, Skyscan NV, Kontich, Belgium). A Gaussian 2D filter was applied to the images before segmentation. Cortical porosity was calculated from tissue volume and pore volume.

\section{Measurement of bone strength}

Bone specimens underwent uniaxial compressive mechanical testing along axis 3, performed with a MTS Criterion Series 40 Electromechanical Universal Test Systems (model C42.503, MTS Corp., Eden Prairie, MN, USA). Specimens were slowly thawed and immersed in $0.9 \% \mathrm{NaCl}$ saline for six hours before testing to ensure full hydration (Zhao et al., 2018). Then, they were subsequently heated and kept at $37^{\circ} \mathrm{C} \pm 0.5^{\circ} \mathrm{C}$ with hydration throughout the compression test by the use of a custom made thermo-regulatory system. The system consisted of a cell filled with saline where the specimen was immersed. A circulation thermostat was used to keep the saline at a stable temperature (Lauda Loop L100, Landa Dr.R. Wobser GMBH \& CO. KG, Germany).

Three preconditioning cycles of $50 \mathrm{~N}$ and a pre-load of $150 \mathrm{~N}$ were applied at a rate of $10^{-4} \mathrm{~S}^{-1}$ to the specimen (Duchemin et al., 2008; Wachter et al., 
2001; Zhao et al., 2018). The specimen was then compressed until failure at a strain rate of $0.1 \mathrm{~s}^{-1}$ to simulate an impact fracture (Carter and Hayes, 1977; Öhman et al., 2011). During the test, displacements of the machine crosshead and load (MTS LSB.503 $5 \mathrm{kN}$ load cell) were registered. The compressive strength, $\sigma_{m}$, was obtained as the maximum stress on the stress-strain curve.

The reproducibility of strength measurement was assessed from a serie of experiments on 15 rectangular parallelepiped shaped specimens with nominal dimensions of $2.5 \mathrm{~mm} \times 3.3 \mathrm{~mm} \times 4.3 \mathrm{~mm}$ cut off the same plate of synthetic bone-mimicking material (Sawbones, Pacific Research Laboratory Inc., Vashon WA, USA). The ultimate strength in all these specimens was assumed to be the same as the plate material was assumed to be homogeneous. This material incorporates short glass fibers which are oriented along the longest dimension of the specimens, which was also the direction along which the specimen was compressed. The same testing protocol as described above was used, except that specimens were not immersed (measurement in air at room temperature). In the 15 specimen group, the strength of one specimen was relatively large (221.0 MPa), it was relatively small for 3 specimens (around 185.6 $\mathrm{MPa} \pm 1.4 \mathrm{MPa}$ ), and the rest of the measured strength fell in a narrow interval around 200.0 MPa with minimum and maximum values of 196.4 and $207.5 \mathrm{MPa}$, respectively. The complete data set is provided as supplementary material. Based on the latter interval, the precision of strength measurement of synthetic bone with our setup was estimated to $6.5 \%$. The reason for the anomalously large or small strength in four specimens was not clearly identified; it may be due to an imperfect alignment of the loading axis or an imperfect geometry. 


\section{Statistics}

The normality of distributions was tested with a Lilliefors test. Comparisons of groups were done with two-sample t-test or alternatively Wilcoxon rank sum test when the data could not be assumed to be normally distributed. The relationships between velocities and porosity, velocities and strength, and strength and porosity were quantified with Spearman's rank correlation coefficients (as some variables were not normally distributed) and modeled with linear regressions. Statistical analyses were performed with MATLAB (The Mathworks Inc., Natick, MA, USA). The level of significance was set to $5 \%$.

\section{Results}

Three representative stress-strain curves from specimens with different density values (25th, median and 75th percentile approximately) are shown in Figure 2. No anomaly in the curve or in the visual appearance of specimens after testing were detected.

For RUS measurement of tibia specimens, between 11 and 22 resonant frequencies (average 15) were measured in the range 140-800 kHz. For femur specimens, between 11 and 28 resonant frequencies (average 18) were measured in the range $60-700 \mathrm{kHz}$. For 5 femur specimens, after solving the inverse problem to determine elastic constants, the misfit error between measured and modeled resonant frequencies was larger than $1 \%$, which is an indication of failure of the measurement (Migliori and Sarrao, 1997). This was possibly due to a misalignment between specimens' orientation and bone material principal directions. These 5 specimens were therefore discarded 


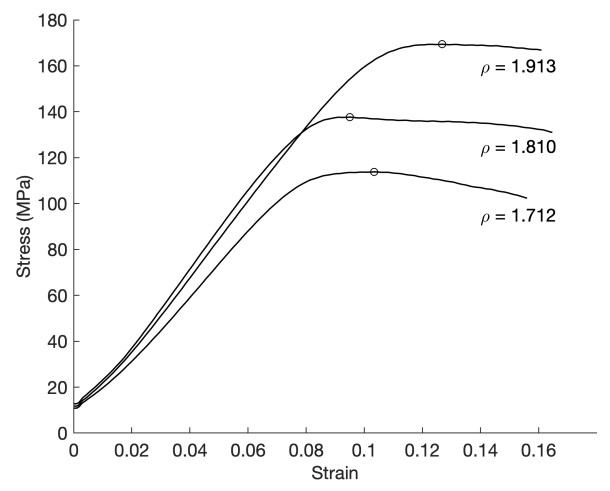

Figure 2: Representative examples of stress-strain compression curves for three specimens with relatively low $\left(\rho=1.712 \mathrm{mg} / \mathrm{mm}^{3}\right)$, intermediate $\left(\rho=1.810 \mathrm{mg} / \mathrm{mm}^{3}\right)$ and high values $\left(\rho=1.913 \mathrm{mg} / \mathrm{mm}^{3}\right)$ of mass density, $\rho$. The circle indicates ultimate stress, $\sigma_{m}$.

from the final analysis. For the rest of the specimens, the mean relative error (standard deviation) between predicted and measured frequencies was $0.71 \%$ $(0.16 \%)$ and $0.47 \%(0.16 \%)$ for tibia and femur, respectively.

A global analysis of the results with data from all measurement modalities led to discard outliers. One femur specimen which included a portion of trabecularized bone from the endosteal interface and with a porosity higher than 30\% was not considered as representative of cortical bone (Bousson et al., 2001) and consequently was not included in the analysis. Velocities of two specimens (one femur and one tibia) were judged to be ouliers (defined as values away from of the median of more than three scaled median absolute deviations); these specimens' data were not included in the analysis. Finally, data were available for further analysis for a set of 15 tibia and 69 femur specimens. Among these, 12 tibia and 38 femur specimens had been subjected to $\mu \mathrm{CT}$ for porosity measurement. Descriptive statistics are provided 
for tibia and femur measurements in Tables 1 and 2, respectively. The data are provided as supplementary material.

Table 1: Descriptive statistics (mean, standard deviation, median, minimal and maximal values) for tibia specimens: $\rho\left(\mathrm{kg} \cdot \mathrm{m}^{-3}\right)$, mass density; $V_{i}(i=1,3)$, longitudinal waves velocities $\left(\mathrm{m} . \mathrm{s}^{-1}\right) ; V_{i}(i=4,6)$, shear waves velocities $\left(\mathrm{m} . \mathrm{s}^{-1}\right) ; \sigma_{m}$, compression strength (MPa); Ct.Po (\%), porosity.

\begin{tabular}{cccccccc}
\hline & $\rho$ & $V_{1}$ & $V_{3}$ & $V_{4}$ & $V_{6}$ & $\sigma_{m}$ & Ct.Po \\
\hline Mean & 1824 & 3054 & 3873 & 1781 & 1478 & 148 & 12.11 \\
SD & 94 & 239 & 90 & 112 & 139 & 27 & 5.21 \\
Median & 1855 & 3103 & 3889 & 1844 & 1544 & 156 & 11.29 \\
$\min$ & 1640 & 2514 & 3704 & 1547 & 1233 & 103 & 6.15 \\
$\max$ & 1960 & 3379 & 4076 & 1920 & 1655 & 179 & 22.82 \\
\hline
\end{tabular}

Table 2: Descriptive statistics (mean, standard deviation, median, minimal and maximal values) for femur specimens : $\rho\left(\mathrm{kg} \cdot \mathrm{m}^{-3}\right)$, mass density; $V_{i}(i=1 \cdots 3)$, longitudinal waves velocities $\left(\mathrm{m} . \mathrm{s}^{-1}\right) ; V_{i}(i=4 \cdots 6)$, shear waves velocities $\left(\mathrm{m} . \mathrm{s}^{-1}\right) ; \sigma_{m}$ (MPa), compression strength; Ct.Po (\%), porosity.

\begin{tabular}{cccccccccc}
\hline & $\rho$ & $V_{1}$ & $V_{2}$ & $V_{3}$ & $V_{4}$ & $V_{5}$ & $V_{6}$ & $\sigma_{m}$ & Ct.Po \\
\hline Mean & 1799 & 3107 & 3150 & 3836 & 1698 & 1659 & 1436 & 143 & 13 \\
SD & 114 & 144 & 149 & 176 & 103 & 124 & 116 & 23 & 5.87 \\
Median & 1819 & 3134 & 3174 & 3855 & 1713 & 1687 & 1458 & 149 & 11.76 \\
$\min$ & 1482 & 2791 & 2838 & 3435 & 1469 & 1393 & 1168 & 95 & 3.33 \\
$\max$ & 1969 & 3367 & 3435 & 4280 & 1890 & 1848 & 1651 & 184 & 30.84 \\
\hline
\end{tabular}

Femur specimens were measured with RUS using an orthotropy framework while a transversely isotropic framework was used for tibia specimens. Orthotropy in femur specimens was nevertheless small: the differences were $1.4 \%$ and $2.4 \%$ between the means of $V_{1}$ and $V_{2}$, and $V_{4}$ and $V_{5}$, respectively. 


$$
(r=-0.74)(\text { Figure 5) }
$$

Table 3: Spearman's rank correlation coefficients between velocities $V_{1}, V_{3}, V_{4}, V_{6}\left(\mathrm{~m} . \mathrm{s}^{-1}\right)$, mass density $\rho\left(\mathrm{kg} \cdot \mathrm{m}^{-3}\right)$, and compression strength $\sigma_{m}$ (MPa), or porosity Ct.Po (\%). The number of specimens used to calculate correlation coefficients with $\sigma_{m}$ is $n=84$ for $\rho, V_{1}, V_{3}$, and $V_{6}$ pooling data from tibia and femur, and $n=69$ for $V_{4}$ considering only femur data. To calculate correlation coefficients with porosity, $n=50$ for $\rho, V_{1}, V_{3}$, and $V_{6}$ pooling data from tibia and femur and $n=38$ for $V_{4}$ considering only femur data. $\mathrm{p}$-value $<10^{-3}$ for all correlations.

\begin{tabular}{lllllll}
\hline & $\rho$ & $V_{1}$ & $V_{3}$ & $V_{4}$ & $V_{6}$ \\
\cline { 2 - 6 }$\sigma_{m}$ & 0.91 & 0.65 & 0.70 & 0.87 & 0.83 \\
Ct.Po & -0.74 & -0.64 & -0.69 & -0.77 & -0.76 \\
\hline
\end{tabular}

281 imens, we averaged $V_{1}$ and $V_{2}$ on the one hand, and $V_{4}$ and $V_{5}$ on the other hand. In the following, $V_{1}$ and $V_{4}$ in femur specimens refer to this average. There was no significant difference between data from tibia and femur specimens except for $V_{4}$. Consequently, for subsequent analyses, we have pooled data from tibia and femur, except for $V_{4}$ for which we present the result of the analyses for femur only (because the number of specimens is much larger than for tibia).

Spearman's rank correlation coefficients between velocities and compression strength, and between velocities and porosity are given in Table 3. All velocities were positively correlated to strength (Figure 3) and negatively correlated to porosity (Figure 4). Strength was negatively correlated to porosity $(r=-0.74)$ (Figure 5).

Linear regression models (Tables 4 and 5) indicate that: i) an increase of strength of $1 \mathrm{MPa}$ is associated to an increase of velocity between $4.32 \mathrm{~ms}^{-1}$

In order to facilitate the comparison between data from femur and tibia spec- 

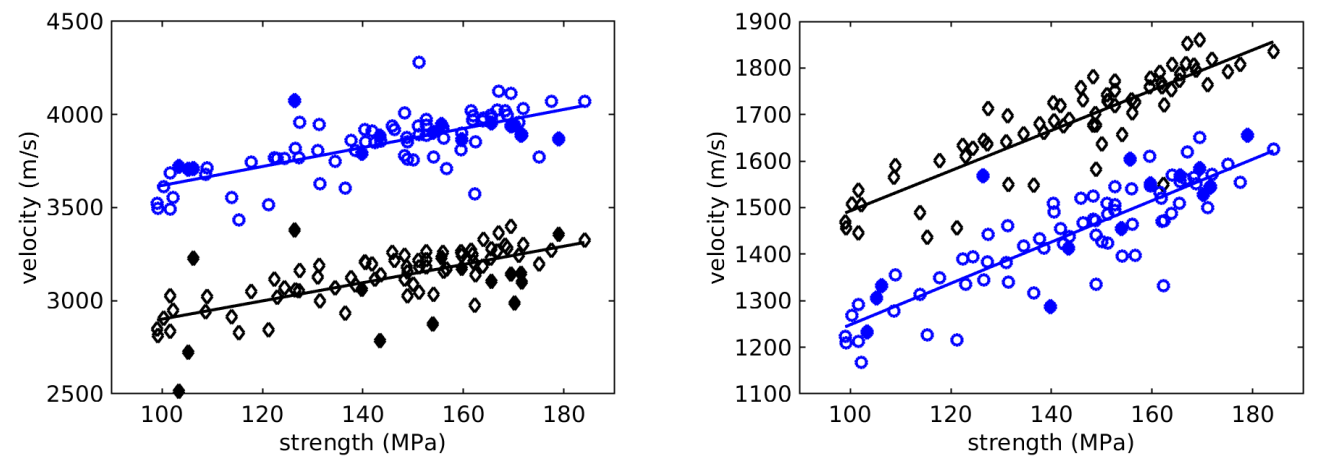

Figure 3: Velocities and compression strength for tibia (filled symbols) and femur specimens, pooled except for $V_{4}$ for which only femur data are shown. Left: longitudinal waves $V_{1}($ black $\diamond)$ and $V_{3}$ (blue $\circ$ ); right: shear waves $V_{4}$ (black $\left.\diamond\right)$ and $V_{6}$ (blue $\circ$ ). The linear regression lines are shown.
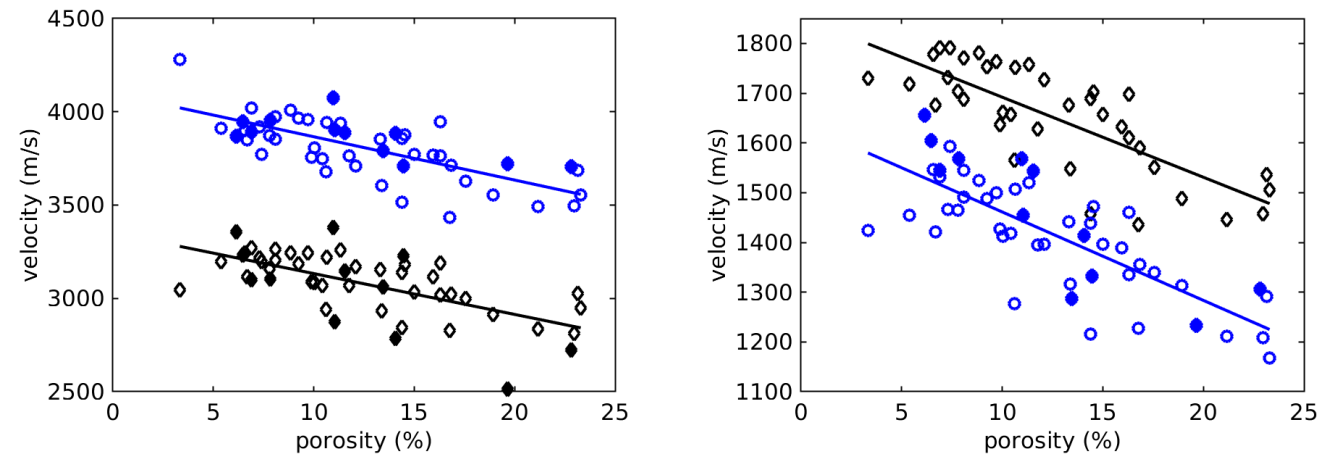

Figure 4: Velocities and porosity for tibia (filled symbols) and femur specimens, pooled except for $V_{4}$ for which only femur data are shown. Left: longitudinal waves $V_{1}$ (black $\diamond$ ) and $V_{3}$ (blue o); right: shear waves $V_{4}$ (black $\left.\diamond\right)$ and $V_{6}$ (blue $\circ$ ). The linear regression lines are shown. 


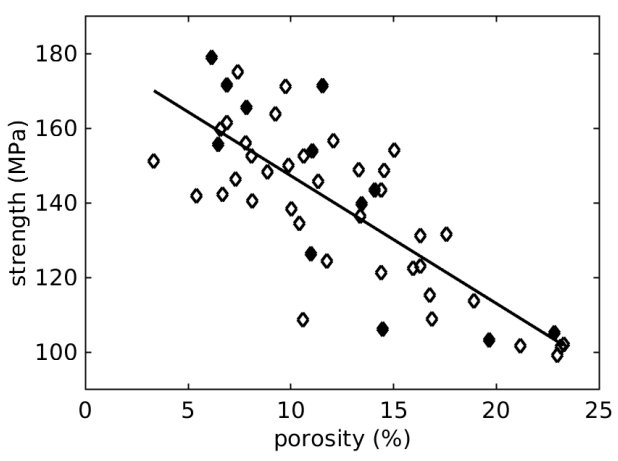

Figure 5: Strength and porosity for tibia (filled symbols) and femur specimens pooled.

and $5.10 \mathrm{~ms}^{-1}$, depending on propagation direction and polarisation ; ii) an increase of porosity of $1 \%$ is associated to a decrease of velocity between $16.1 \mathrm{~ms}^{-1}$ and $23.2 \mathrm{~ms}^{-1}$, depending on propagation direction and polarisation. The sensitivity of the different velocities to changes of strength or porosity can be expressed in terms of percentage of change of each velocity: for an increase of strength of $1 \mathrm{MPa}, V_{1}, V_{3}, V_{4}$, and $V_{6}$ increase $0.16 \%$, $0.13 \%, 0.25 \%$, and $0.31 \%$, respectively, suggesting a higher sensitivity of shear velocities to changes of strength. For an increase of porosity of $1 \%, V_{1}$, $V_{3}, V_{4}$, and $V_{6}$ decrease $0.70 \%, 0.60 \%, 0.95 \%$, and $1.23 \%$, respectively, also suggesting a higher sensitivity of shear velocities to changes of porosity.

\section{Discussion}

In this study, US velocities, compression strength and porosity in cortical bone specimens of tibia and femur of elderly human donors were measured. We believe our data are representative of the elderly population as the range of mass density and porosity of our specimens span the physiological range as documented in other studies involving large collections of specimens (Rho 
Table 4: Linear models of velocities, $V_{1}, V_{3}, V_{4}, V_{6}\left(\mathrm{~ms}^{-1}\right)$ as a function of strength, $\sigma_{m}$ (MPa). RMSE is the root-mean-square-error. p-value $<10^{-3}$ for all models.

\begin{tabular}{lcc} 
Model & RMSE & $R^{2}$ \\
\hline$V_{1}=2412+4.89 \sigma_{m}$ & 121 & 0.46 \\
$V_{3}=3109+5.10 \sigma_{m}$ & 115 & 0.50 \\
$V_{4}=1061+4.32 \sigma_{m}$ & 55 & 0.75 \\
$V_{6}=804+4.44 \sigma_{m}$ & 61 & 0.73 \\
\hline
\end{tabular}

Table 5: Linear models of velocities, $V_{1}, V_{3}, V_{4}, V_{6}\left(\mathrm{~ms}^{-1}\right)$ as a function of porosity, Ct.Po (\%). RMSE is the root-mean-square-error. p-value $<10^{-3}$ for all models.

\begin{tabular}{lcc}
\hline Model & RMSE & $R^{2}$ \\
\hline$V_{1}=3352-21.9$ Ct.Po & 134 & 0.42 \\
$V_{3}=4099-23.2$ Ct.Po & 117 & 0.51 \\
$V_{4}=1853-16.1$ Ct.Po & 69 & 0.60 \\
$V_{6}=1639-17.8$ Ct.Po & 75 & 0.60 \\
\hline
\end{tabular}

et al., 1995; Bousson et al., 2001). Results showed that velocities of US waves of shear and longitudinal polarisation, propagating along or perpendicular to osteons, are correlated to compressive strength and porosity. Roughly, according to the linear regression models, a decrease of velocity of $100 \mathrm{~ms}^{-1}$ corresponds to a loss of $20 \mathrm{MPa}$ of strength (which is approximately $10 \%$ of maximum strength value observed) and to an increase of porosity of $5 \%$. There is a trend of a higher sensitivity of shear wave velocities to changes of strength and porosity, compared to longitudinal wave velocities. This trend for porosity is consistent with simulation data (Baron et al., 2007) conducted for plane waves centered at $1 \mathrm{MHz}$. 
To the best of our knowledge, the data presented in this study is the first quantification of the relationship between bulk wave US velocities in cortical bone and strength. The result that velocities and strength are correlated was nevertheless expected because velocity is known to be related to porosity and porosity is related to strength (Eneh et al., 2016; Mirzaali et al., 2016). The negative correlations between both shear and longitudinal wave velocities and porosity were also previously demonstrated in a simulation study conducted on plane waves centered at $1 \mathrm{MHz}$ (Baron et al., 2007). Empirically, only the longitudinal wave velocity has been previously considered (Grondin et al., 2012; Mathieu et al., 2013; Eneh et al., 2016). Porosity variation was found to explain about $30 \%$ of the variation of velocity along osteons (Grondin et al., 2012; Mathieu et al., 2013) and about 50\% of the variation of velocity perpendicular to osteons (Eneh et al., 2016). The correlation coefficients between longitudinal wave velocities and porosity from the present study are in good agreement with the latter. Finally, the correlation found between strength and porosity in this study (Figure $5, \mathrm{r}=-0.74$ ) is also consistent with the results from previous experimental studies in human cortical bone (Boughton et al., 2019; Mirzaali et al., 2016).

One originality of our protocol was to use RUS to determine bulk wave velocities. RUS has provided the full transversely isotropic stiffness tensor of tibia specimens and the full orthotropic stiffness tensor of femur specimens. In this study, we have presented the analysis for the velocities of waves propagating along the radial, circumferential and axial directions of bone. The velocities of waves propagating along other directions can be calculated with the data provided as supplementary material. A specificity of RUS, com- 
pared, e.g., to time-of-flight techniques for velocity measurement (Peralta et al., 2017), is that shear moduli are obtained with an intrinsically higher precision compared to longitudinal moduli (Migliori and Sarrao, 1997; Cai et al., 2017). This may be a reason why correlation coefficients with strength and porosity are higher for shear velocities compared to those with longitudinal velocities. We made the choice to measure compression strength on the same specimens used for RUS rather than using other specimens with a dedicated shape prepared, e.g., from adjacent locations in the diaphysis. This made it possible to get rid of the variations of bone properties (heterogeneity) along the diaphysis which may have had the effect of decreasing the correlations between strength and velocities.

The correlations between velocities and porosity and between velocities and strength are only moderate. One possible explanation is that other factors than porosity affect velocities. As the extravascular matrix mineral content (not measured in the present study) is known to explain a part of the variations of elastic properties after adjusting for porosity (Cai et al., 2019), the inter-specimen variations of mineral content may explain a part of velocity variations not captured in the present study. Similarly, our results suggest that the inter-specimen variations of some properties affecting strength are not captured by the measurement of velocities. Bone resistance to crack propagation is related to extravascular matrix heterogeneity, mineralization, collagen properties and the mechanical behavior of mineralized collagen fibers at strain levels far beyond those involved in US propagation (Zimmermann et al., 2015). Finally, we have used porosity, e.g., pore volume fraction, to characterize the effect of the vascular pore network on velocities 
and strength. The shape, size, and distribution of porosities and the presence of large pores in some samples may also affect strength (Iori et al., 2019). In this study, we have not computed other parameters of the pore network such as mean cortical pore volume and mean cortical pore diameter because of the limited precision of the estimation of these parameters from conventional micro-CT subjected to beam hardening and cone beam reconstruction artifacts (Ostertag et al., 2016).

Quantitative US methods to assess bone health could take advantage of measuring velocities in cortical bone. Lee et al. (1997) showed that speed of sound measured with a low frequency $(250 \mathrm{kHz})$ axial transmission method at the tibia was highly correlated $\left(R^{2}=0.75\right)$ with strength measured in tension. However, this axial transmission modality measured the speed of sound of a guided wave which not only depends on bulk wave velocities but also on cortical bone thickness. Our study suggests that all bulk wave velocities (with different directions and polarisations) are worth measuring in vivo as they carry information on bone strength. Based on the linear regression models with strength, the range of variation of velocities is in the order of $400 \mathrm{~m} . \mathrm{s}^{-1}$ and $350 \mathrm{~m} . \mathrm{s}^{-1}$ for longitudinal and shear waves, respectively. These numbers should be compared with the precision of US devices designed for clinical use. For instance, the axial transmission technique provides the velocity of the first arriving signal, a quantity representative of bulk longitudinal wave velocity $V_{3}$ for a thick bone (Bossy et al., 2002), with a precision of $\pm 20 \mathrm{~m} \cdot \mathrm{s}^{-1}$ in vivo (inter-operator reproducibility using the clinical protocol) (Talmant et al., 2009). In a pilot study on two healthy volunteers using an array probe for imaging of tibia and radius cortex, Renaud et al. (2018) 
reported the precision of longitudinal wave velocities assessment based on the standard deviation of 5 measurements with repositioning, which was between 40 and $140 \mathrm{~m} \cdot \mathrm{s}^{-1}$ for $V_{1}$ and between 50 and $70 \mathrm{~m} \cdot \mathrm{s}^{-1}$ for $V_{3}$. We conclude that differences in bone mechanical quality reflected in velocities could actually be probed in vivo as the range of inter-specimen variation of velocities is close to an order of magnitude larger than the precision of in vivo devices.

Finally, this study has some limitations. Bone specimens were collected at two skeletal site (femoral and tibial diaphysis) of bones from elderly donors without documentation on the existence of bone pathologies. Therefore, the findings in this work may not apply to other bone sites, age groups, or bone with pathologies. However, we found little differences in the measured variables between tibia and femur, suggesting that our conclusions may be valid for most cortical bone sites. The shape of the specimens, which had a relatively small aspect ratio (about 1.4 and 1.6 for femur and tibia specimens, respectively) was dictated by the requirements of RUS technique (Migliori and Sarrao, 1997; Bernard et al., 2013) to maximize the sensitivity of resonant frequencies to all elastic coefficients. This made the strength measurement configuration sub-optimal as the artifacts due to friction on the compression platens decrease with aspect ratio (Keaveny et al., 1993). In addition, imperfections in the geometrical shape of the samples, i.e. imperfect rectangular parallelepiped samples, could also cause errors in the measured values of strength. We tried to minimize friction by using polished platens and the protocol was extensively tested on reference materials from which we estimated the precision of strength measurement to $6.5 \%$. The protocol accuracy 
was not estimated and further studies are needed to quantify the systematic error in strength measurements.

To conclude, this study evidences that all US velocities reflect strength and porosity of cortical bone. The data provide a rationale for the measurement of one or several velocities in vivo as a biomarker of bone health. Measuring velocities in vivo can be achieved, e.g. with axial transmission (Foiret et al., 2014) or quantitative imaging (Renaud et al., 2018; Nguyen Minh et al., 2020) and can complement US diagnosis of cortical bone fragility.

\section{Supplementary material}

A file SuppMaterial.xls is provided which contains: i) the data used for the statistical analysis (strength, porosity, density and elastic coefficients) ii) the data used to assess the reproducibility of strength measurement with synthetic bone material.

\section{Acknowledgment}

The authors would like to thank Marwa Hammami, Pascal Dargent and Noémie Taupin for specimen preparation and the help in conducting mechanical tests. This work was supported by grants from the Deutsche Forschungsgemeinschaft (DFG Ra1380/9-1) and by the Agence Nationale de la Recherche (ANR-14-CE35-0030-01) within the TaCo-Sound project. 


\section{References}

Ahmed LA, Shigdel R, Joakimsen RM, Eldevik OP, Eriksen EF, GhasemZadeh A, Bala Y, Zebaze R, Seeman E, Bjørnerem A. Measurement of cortical porosity of the proximal femur improves identification of women with nonvertebral fragility fractures. Osteoporosis International, 2015;26:21372146.

Auld B. Acoustic fields and waves in solids, 1975.

Bala Y, Zebaze R, Seeman E. Role of cortical bone in bone fragility. Current Opinion in Rheumatology, 2015;27:406-413.

Baron C, Talmant M, Laugier P. Effect of porosity on effective diagonal stiffness coefficients (cii) and elastic anisotropy of cortical bone at 1MHz: A finite-difference time domain study. The Journal of the Acoustical Society of America, 2007;122:1810-1817.

Bernard S, Grimal Q, Laugier P. Accurate measurement of cortical bone elasticity tensor with resonant ultrasound spectroscopy. Journal of the Mechanical Behavior of Biomedical Materials, 2013;18:12-19.

Bernard S, Grimal Q, Laugier P. Resonant ultrasound spectroscopy for viscoelastic characterization of anisotropic attenuative solid materials. The Journal of the Acoustical Society of America, 2014;135:2601-13.

Bernard S, Marrelec G, Laugier P, Grimal Q. Bayesian normal modes identification and estimation of elastic coefficients in resonant ultrasound spectroscopy. Inverse Problems, 2015;31:065010. 
Bernard S, Schneider J, Varga P, Laugier P, Raum K, Grimal Q. Elasticitydensity and viscoelasticity-density relationships at the tibia mid-diaphysis assessed from resonant ultrasound spectroscopy measurements. Biomechanics and modeling in mechanobiology, 2016;15:97-109.

Bossy E, Talmant M, Laugier P. Effect of bone cortical thickness on velocity measurements using ultrasonic axial transmission: A 2D simulation study. Journal of Acoustical Society of America, 2002;112:297-307.

Boughton OR, Ma S, Cai X, Yan L, Peralta L, Laugier P, Marrow J, Giuliani F, Hansen U, Abel RL, Grimal Q, Cobb JP. Computed tomography porosity and spherical indentation for determining cortical bone millimetre-scale mechanical properties. Scientific Reports, 2019;9:7416.

Bousson V, Meunier A, Bergot C, Vicaut E, Rocha MA, Morais MH, LavalJeantet AM, Laredo JD. Distribution of intracortical porosity in human midfemoral cortex by age and gender. Journal of Bone and Mineral Research, 2001;16:1308-1317.

Briot K, Paternotte S, Kolta S, Eastell R, Felsenberg D, Reid DM, Glüer CC, Roux C. FRAX®: Prediction of major osteoporotic fractures in women from the general population: The OPUS study. PLoS ONE, 2013;8.

Cai X, Follet H, Peralta L, Gardegaront M, Farlay D, Gauthier R, Yu B, Gineyts E, Olivier C, Langer M, Gourrier A, Mitton D, Peyrin F, Grimal Q, Laugier P. Anisotropic elastic properties of human femoral cortical bone and relationships with composition and microstructure in elderly. Acta Biomaterialia, 2019;90:254-266. 
Cai X, Peralta L, Gouttenoire PJ, Olivier C, Peyrin F, Laugier P, Grimal Q. Quantification of stiffness measurement errors in resonant ultrasound spectroscopy of human cortical bone. The Journal of the Acoustical Society of America, 2017;142:2755-2765.

Carter DR, Hayes WC. The compressive behavior of bone as a two-phase porous structure. J. Bone Joint Surg., 1977;59:954-962.

Cooper C, Ferrari SL. IOF compendium of osteoporosis. Tech. rep., 2017.

Duchemin L, Bousson V, Raossanaly C, Bergot C, Laredo JD, Skalli W, Mitton D. Prediction of mechanical properties of cortical bone by quantitative computed tomography. Medical Engineering and Physics, 2008;30:321-328.

Eneh CT, Malo MK, Karjalainen JP, Liukkonen J, Töyräs J, Jurvelin JS. Effect of porosity, tissue density, and mechanical properties on radial sound speed in human cortical bone. Medical Physics, 2016;43:2030-2039.

Espinoza Orías AA, Deuerling JM, Landrigan MD, Renaud JE, Roeder RK. Anatomic variation in the elastic anisotropy of cortical bone tissue in the human femur. Journal of the Mechanical Behavior of Biomedical Materials, $2009 ; 2: 255-263$.

Foiret J, Minonzio JG, Chappard C, Talmant M, Laugier P. Combined estimation of thickness and velocities using ultrasound guided waves: A pioneering study on in vitro cortical bone samples. IEEE Transactions on Ultrasonics, Ferroelectrics, and Frequency Control, 2014;61:1478-1488.

Granke M, Grimal Q, Saïed A, Nauleau P, Peyrin F, Laugier P. Change in 
porosity is the major determinant of the variation of cortical bone elasticity at the millimeter scale in aged women. Bone, 2011;49:1020-1026.

Grimal Q, Laugier P. Quantitative Ultrasound Assessment of Cortical Bone Properties Beyond Bone Mineral Density, 2019;40:16-24.

Grondin J, Grimal Q, Yamamoto K, Matsukawa M, Saïed A, Laugier P. Relative contributions of porosity and mineralized matrix properties to the bulk axial ultrasonic wave velocity in human cortical bone. Ultrasonics, 2012;52:467-471.

Holzer G, Von Skrbensky G, Holzer LA, Pichl W. Hip fractures and the contribution of cortical versus trabecular bone to femoral neck strength. Journal of Bone and Mineral Research, 2009;24:468-474.

Hudelmaier M, Kuhn V, Lochmüller E, Well H, Priemel M, Link T, Eckstein F. Can geometry-based parameters from pqct and material parameters from quantitative ultrasound (qus) improve the prediction of radial bone strength over that by bone mass (dxa)? Osteoporosis international, 2004;15:375-381.

Iori G, Peralta L, Reisinger A, Heyer F, Wyers C, van den Bergh J, Pahr D, Raum K. Femur strength predictions by nonlinear homogenized voxel finite element models reflect the microarchitecture of the femoral neck. Medical Engineering \& Physics, 2020;79:60-66.

Iori G, Schneider J, Reisinger A, Heyer F, Peralta L, Wyers C, Gräsel M, Barkmann R, Glüer CC, van den Bergh J, et al. Large cortical bone 
pores in the tibia are associated with proximal femur strength. PloS one, 2019;14:e0215405.

Karjalainen J, Riekkinen O, Töyräs J, Kröger H, Jurvelin J. Ultrasonic assessment of cortical bone thickness in vitro and in vivo. IEEE Transactions on Ultrasonics, Ferroelectrics, and Frequency Control, 2008;55:2191-2197.

Keaveny TM, Borchers RE, Gibson LJ, Hayes WC. Theoretical analysis of the experimental artifact in trabecular bone compressive modulus. Journal of biomechanics, 1993;26:599-607.

Laugier P, Haïat G. Bone quantitative ultrasound, 2011.

Lee SC, Coan BS, Bouxsein ML. Tibial ultrasound velocity measured in situ predicts the material properties of tibial cortical bone. Bone, 1997;21:11925.

Leisure RG, Willis F. Resonant ultrasound spectroscopy. Journal of Physics: Condensed Matter, 1997;9:6001.

Mathieu V, Chappard C, Vayron R, Michel A, Haïat G. Radial anatomic variation of ultrasonic velocity in human cortical bone. Ultrasound in Medicine and Biology, 2013;39:2185-2193.

Mayhew PM, Thomas CD, Clement JG, Loveridge N, Beck TJ, Bonfield W, Burgoyne CJ, Reeve J. Relation between age, femoral neck cortical stability, and hip fracture risk. Lancet, 2005;366:129-135.

Migliori A, Sarrao JL. Resonant ultrasound spectroscopy. Wiley, New York, 1997. 
Minonzio JG, Bochud N, Vallet Q, Ramiandrisoa D, Etcheto A, Briot K, Kolta S, Roux C, Laugier P. Ultrasound-Based Estimates of Cortical Bone Thickness and Porosity Are Associated With Nontraumatic Fractures in Postmenopausal Women: A Pilot Study. Journal of Bone and Mineral Research, 2019;34:1585-1596.

Mirzaali MJ, Schwiedrzik JJ, Thaiwichai S, Best JP, Michler J, Zysset PK, Wolfram U. Mechanical properties of cortical bone and their relationships with age, gender, composition and microindentation properties in the elderly. Bone, 2016;93:196-211.

Nguyen Minh H, Du J, Raum K. Estimation of Thickness and Speed of Sound in Cortical Bone Using Multifocus Pulse-Echo Ultrasound. IEEE Transactions on Ultrasonics, Ferroelectrics, and Frequency Control, 2020;67:568579 .

Öhman C, Baleani M, Pani C, Taddei F, Alberghini M, Viceconti M, Manfrini M. Compressive behaviour of child and adult cortical bone. Bone, 2011;49:769-776.

Orías AAE, Deuerling JM, Landrigan MD, Renaud JE, Roeder RK. Anatomic variation in the elastic anisotropy of cortical bone tissue in the human femur. Journal of the mechanical behavior of biomedical materials, 2009;2:255-263.

Ostertag A, Peyrin F, Gouttenoire PJ, Laredo JD, Devernejoul MC, Cohen Solal M, Chappard C. Multiscale and multimodality computed tomography 
for cortical bone analysis. Physics in Medicine and Biology, 2016;61:85538576.

Peralta L, Cai X, Laugier P, Grimal Q. A critical assessment of the invitro measurement of cortical bone stiffness with ultrasound. Ultrasonics, 2017;80:119-126.

Raum K, Grimal Q, Varga P, Barkmann R, Glüer CC, Laugier P. Ultrasound to assess bone quality. Curr Osteoporos Rep, 2014;12:154-162.

Raum K, Leguerney I, Chandelier F, Bossy E, Talmant M, Saied A, Peyrin F, Laugier P. Bone microstructure and elastic tissue properties are reflected in QUS axial transmission measurements. Ultrasound in Medicine and Biology, 2005;31:1225-35.

Renaud G, Kruizinga P, Cassereau D, Laugier P. In vivo ultrasound imaging of the bone cortex. Physics in Medicine and Biology, 2018;63:125010.

Rho J, Hobathot MC, Ashman R. Relations of mechanical properties to densi $\eta$ and CT numbers in human bone. Medical Engineering \& Physics, $1995 ; 17: 347-355$.

Rho JY. An ultrasonic method for measuring the elastic properties of human tibial cortical and cancellous bone. Ultrasonics, 1996;34:777-783.

Sai H, Iguchi G, Tobimatsu T, Takahashi K, Otani T, Horii K, Mano I, Nagai I, Iio H, Fujita T, Yoh K, Baba H. Novel ultrasonic bone densitometry based on two longitudinal waves: Significant correlation with pQCT measurement values and age-related changes in trabecular bone density, 
cortical thickness, and elastic modulus of trabecular bone in a normal Japanese po. Osteoporosis International, 2010;21:1781-1790.

Schneider J, Iori G, Ramiandrisoa D, Hammami M, Gräsel M, Chappard C, Barkmann, Reinhard Laugier P, Grimal Q, Minonzio, Jean-Gabriel Raum K. Ex vivo cortical porosity and thickness predictions at the tibia using ultrasonic guided waves. Archives of Osteoporosis, 2019;14:1-11.

Siris ES, Chen YT, Abbott TA, Barrett-Connor E, Miller PD, Wehren LE, Berger ML. 128 Bone Mineral Density Thresholds for Pharmacological Intervention to Prevent Fractures. Archives of internal medicine, 2004;164:1108-1112.

Talmant M, Kolta S, Roux C, Haguenauer D, Vedel I, Cassou B, Bossy E, Laugier P. In vivo performance evaluation of bi-directional ultrasonic axial transmission for cortical bone assessment. Ultrasound Med Biol, 2009;35:912-919.

Wachter NJ, Augat P, Krischak GD, Sarkar MR, Mentzel M, Kinzl L, Claes L. Prediction of strength of cortical bone in vitro by microcomputed tomography. Clin Biomech, 2001;16:252-256.

Zebaze R, Libanati C, McClung MR, Zanchetta JR, Kendler DL, Høiseth A, Wang A, Ghasem-Zadeh A, Seeman E. Denosumab Reduces Cortical Porosity of the Proximal Femoral Shaft in Postmenopausal Women With Osteoporosis. Journal of Bone and Mineral Research, 2016;31:1827-1834.

Zebaze RM, Ghasem-Zadeh A, Bohte A, Iuliano-Burns S. Intracortical Re- 
606 modelling and Porosity in the Distal Radius and Post-mortems Femurs of 607 Women: A Cross Sectional Study. Lancet, 2010;375:1729-36.

608

609

610

611

612

Zhao S, Arnold M, Abel RL, Cobb JP, Ma S, Hansen U, Boughton O. Standardizing compression testing for measuring the stiffness of human bone. Bone and Joint Research, 2018;7:524-538.

Zimmermann EA, Busse B, Ritchie RO. The fracture mechanics of human bone: influence of disease and treatment. BoneKEy Reports, 2015;4:743. 